\title{
红壤可变电荷矿物的酸碱缓冲能力及表面络合模型
}

\author{
程鹏飞 $a, b$ 王莹 ${ }^{b}$ 程宽 ${ }^{b}$ 李芳柏 ${ }^{b}$ 秦好丽 ${ }^{a}$ 刘同旭*, $b$

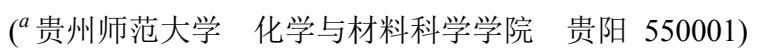 \\ $\left({ }^{b}\right.$ 广东省生态环境技术研究所 广东省农业环境综合治理重点实验室 广州 510650)
}

\begin{abstract}
摘要 氧化铁和高岭石是红壤中可变电荷的主要来源, 对红壤的酸碱变化起到缓冲作用. 本研究基于红壤矿物的表征 和酸碱滴定实验结果, 采用 1 -site $/ 2-\mathrm{p} K$ 表面络合模型获得了其表面活性位点浓度 $H_{\mathrm{s}}$ 、密度 $D_{\mathrm{s}}$ 、酸碱平衡常数 $\mathrm{p} K_{\mathrm{a}}^{\mathrm{int}}$ 以 及电荷零点 $\mathrm{pH}_{\mathrm{pzc}}$ 等相关参数, 定量解析了氧化铁和高岭土的酸碱缓冲能力. 结果表明: 该模型能较好地适用于分析针 铁矿、赤铁矿及高岭石的表面酸碱性质; 针铁矿、高岭石表面活性位点浓度 $H_{\mathrm{s}}$ 较高, 说明其对酸具有较好的缓冲效果. 根据上述酸碱性质参数, 模拟计算了不同 $\mathrm{pH}$ 下的矿物表面化学物种, 揭示了矿物表面反应缓冲土壤酸碱变化的机制. 采用上述酸碱滴定方法及模型计算方法, 分析实际林地红壤样品的酸碱缓冲能力, 并采用表面络合模型计算了其表面 化学物种, 验证了该方法用于林地红壤酸碱缓冲能力分析的可行性.

关键词＼cjkstart氧化铁; 高岭石; 红壤; 酸碱缓冲能力; 表面络合模型
\end{abstract}

\section{The Acid-Base Buffer Capacity of Red Soil Variable Charge Minerals and Its Surface Complexation Model}

\author{
Cheng, Pengfei ${ }^{a, b} \quad$ Wang, Ying $^{b} \quad{\text { Cheng, } \text { Kuan }^{b} \quad \text { Li, Fangbai }}^{b} \quad$ Qin, Haoli ${ }^{a}$ \\ Liu, Tongxu*,b \\ ( ${ }^{a}$ School of Chemistry and Materials Science, Guizhou Normal University, Guiyang 550001, China) \\ ( ${ }^{b}$ Guangdong Institute of Eco-environmental Science \& Technology, Guangdong Key Laboratory of Integrated \\ Agro-environmental Pollution Control and Management, Guangzhou 510650, China)
}

\begin{abstract}
Iron oxides and kaolinite are the main sources of variable charges in the red soil. As a result of being protonated and deprotonated under different acid-base conditions, the surface hydroxyl groups can buffer the $\mathrm{pH}$ changes of red soil. In this study, iron oxide and kaolinite were titrated by the standard $\mathrm{HCl}$ and $\mathrm{NaOH}$ solution through the auto potentiometric titration under the controlled $\mathrm{pH}=2.9 \sim 9.5$, to study the surface charge of soil minerals. The X-ray diffraction (XRD), Fourier transform infrared spectroscopy (FTIR), and $\mathrm{N}_{2}$ desorption/adsorption isotherms (BET) were used to characterize the crystal structures, surface groups and specific surface areas of soil minerals. Based on the characterization data and titration curves, the acid-base properties of the minerals were analyzed by using 1-site/2-pK surface complexation model. The Gran plot method, commonly used to determine the equivalence points, was applied to calculate the concentration $\left(H_{\mathrm{s}}\right)$ and density $\left(D_{\mathrm{s}}\right)$ of the surface active sites on the soil minerals. The acid-base equilibrium constants $\left(\mathrm{p} K_{\mathrm{a}}^{\text {int }}\right)$ of soil minerals were obtained by extrapolation and the corresponding $\mathrm{pH}_{\mathrm{pzc}}$ were calculated by the following formula: $\mathrm{pH}_{\mathrm{pzc}}=1 / 2\left(\mathrm{p} K_{\mathrm{a} 1}{ }^{\text {int }}+\mathrm{p} K_{\mathrm{a} 2}{ }^{\text {int }}\right)$. The result of calculated value of $\mathrm{pH}_{\mathrm{pzc}}$ was nearly equal with the experimental value, which showed that it is feasible to apply this model calculation method on the soil minerals. In addition, the above parameters can explain the acid-base buffer capacity of the minerals quantitatively. The results show that goethite and kaolinite have the higher surface active site concentration. According to the parameters, the surface chemical speciation of minerals at different $\mathrm{pH}$ were calculated by Visual Minteq software with the double layer model (DLM) to explain the mechanism of acid-base buffer behavior on the mineral surfaces. Finally, the acid-base titration method and model calculation approach were also used to analyze the acid-base buffer capacity of the natural red soil samples. The feasibility of this method on the red soil was further verified. Then, the surface chemical species $\left(\equiv \mathrm{SOH}_{2}{ }^{+}, \equiv \mathrm{SO}^{-}\right.$and $\equiv \mathrm{SOH}$ ) of the red soil were calculated by surface complex model to further explain their acid-base buffer mechanism.

Keywords iron oxide; kaolinite; red soil; acid-base buffer capacity; surface complexation model
\end{abstract}

\section{1 引言}

我国热带亚热带地区，广泛分布着各种不同类型的 红壤, 包括砖红壤、赤红壤、红壤、黄壤, 统称为红壤
系列. 红壤是我国南方 14 省(区)的重要土壤类型和耕作

土壤，总面积约 218 万平方公里，占全国总土地面积的 $22.7 \%$ ，占全国耕地面积的 $28 \%^{[1,2]}$. 但目前红壤酸化日

* E-mail: txliu@soil.gd.cn; Tel.: 020-87025180

Received February 15, 2017; published April 14, 2017.

Project supported by the "973" Program (2014CB441002) and the National Natural Science Foundation of China (41571130052).

项目受科技部红壤酸化 973 项目(2014CB441002)和国家自然科学基金委中英国际合作红壤关键带项目(41571130052)资助. 
益严重, 造成土壤中 $\mathrm{Ca}^{+} 、 \mathrm{Mg}^{2+} 、 \mathrm{~K}^{+}$等盐基性养分离子 大量淋失 ${ }^{[3]}, \mathrm{H}^{+}$、铝和镉等毒性元素浓度增加、活性增 强 ${ }^{[4,5]}$, 严重影响了当地农业生产和生态平衡. 酸沉降、 不合理的农业措施等人为因素是造成土壤酸化主要原

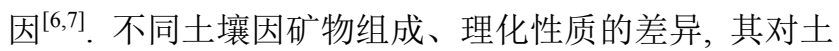
壤酸化的缓冲能力也不尽相同 ${ }^{[8]}$, 因此土壤自身性质也 是影响土壤酸化程度的原因之一. 我国参考欧美等发达 地区酸化研究模式 ${ }^{[9,10]}$ 在土壤酸化及调控方面已取得很 多重要进展 ${ }^{[11,12]}$. 但欧美等发达地区地处温带, 土壤以 恒电荷土壤为主. 而我国酸化严重的热带、亚热带地区 红壤, 以可变电荷土壤为主, 在矿物组成、理化性质等 方面都不同于温带恒电荷土壤. 所以有必要针对我国红 壤区的矿物类型和酸化特征, 建立相应的酸化评价模 式 ${ }^{[13]}$.

我国红壤的矿物组成随红壤形成的脱硅富铝化过 程而变化. 在高温多雨条件下, 土壤中硅酸盐类矿物强 烈分解, 硅和盐基淋失, 铁铝等氧化物明显聚积、粘粒 与次生矿物不断形成. 因此, 脱硅富铝化后的红壤矿物 主要包括粘粒矿物和氧化物, 粘粒矿物以高岭石为主, 含有极少蒙脱石或伊利石, 氧化物主要包括氧化铁、氧 化铝、氧化硅 ${ }^{[14 ~ 16]}$. 其中氧化铁以赤铁矿、针铁矿为主, 水铁矿在红壤中也有分布但不稳定, 在干燥的热带、亚 热带气候下转变为赤铁矿, 在潮湿的温带气候下转变为 针铁矿 ${ }^{[14]}$. 红壤由于氧化铁含量不同呈现不同颜色, 因 此红壤系列分为砖红壤、赤红壤、红壤、黄壤. 一般赤 铁矿愈多色愈红, 针铁矿等水化氧化铁愈多, 粒子愈细, 土色愈黄 ${ }^{[17]}$. 不同母质发育的红壤所含氧化铁种类和 含量也不同. 王旭刚等 ${ }^{[18,19]}$ 报道, 广东博罗县玄武岩发 育赤红壤和砖红壤粘土矿物以高岭石为主, 且含有针铁 矿和赤铁矿, 其中赤红壤以针铁矿为主, 砖红壤以赤铁 矿为主. 广东南雄第四纪红粘土发育红壤也同时有针铁 矿、赤铁矿存在. 李庆逵等 ${ }^{[1]}$ 报道广东博罗花岗岩发育 黄壤中氧化铁主要为针铁矿, 海南那大镇片麻岩发育砖 红壤也含有大量针铁矿. 此外, 土壤脱硅富铝化过程除 高岭石、氧化铁生成外, 也会有铝氧化物聚积. 主要以 三水铝石形式存在于风化程度较高的砖红壤中, 与前两 者相比含量较少, 分布较局限 ${ }^{[1,16,17]}$.

高岭石及铁铝氧化物的形成使得土壤表面具有了 不同的电荷性质. 高岭石与蒙脱石相比, 其表面永久电 荷极少, 但含有较多的活性硅、铝羟基, 可以接受土壤 溶液中质子转变为表面正电荷 ${ }^{[20]}$. 针铁矿、赤铁矿在湿 润条件下易水化使表面带有铁羟基，也能接受质子带有 正电荷 ${ }^{[16,21,22]}$. 这种表面电荷变化能够降低土壤环境中 的质子量, 所以高岭石、氧化铁以及富含它们的自然红 壤对酸化均具有一定的缓冲能力 ${ }^{[20 ~ 22]}$. 关于缓冲能力 的研究, 欧美等地采用阳离子交换量 CEC 表示表面负 电荷量, 并建立了临界负荷图表示不同土壤对酸化的敏 感性, 但这些方法更适用于恒电荷土壤 ${ }^{[13,23,24]}$. 酸碱电
位滴定法实现了对表面可变电荷的测定，但单纯的表面 可变电荷量并不足以描述土壤表面酸碱的缓冲能力及 其机制. 目前, 有较多研究表明 ${ }^{[25,26]}$, 金属氧化物与离 子络合反应的表面络合模型(SCM)能够获得相关络合常 数以描述氧化物的表面酸碱性质, 并较好地解释界面反 应机制. 由于金属氧化物是红壤可变电荷矿物中的重要 组成部分, 关于 SCM 在氧化铁表面络合反应的应用已 有报道 ${ }^{[20,27]}$, 但是此 SCM 是否也适用于土壤矿物以及 自然红壤表面酸碱性质的分析, 仍需进一步研究. 本研 究则尝试将电位滴定法与 SCM 结合用于测定红壤的主 要矿物包括高岭石、针铁矿、赤铁矿以及红壤样品的酸 碱缓冲能力, 并通过表面化学物种模拟对其酸碱缓冲机 制进行探讨, 以期能够建立专门针对红壤酸碱缓冲能力 测试的方法, 帮助理解红壤自身对酸化的缓冲机制, 为 我国红壤酸化预测和阻控提供依据.

\section{2 结果与讨论}

\section{1 矿物表征结果}

三种可变电荷矿物的 $\mathrm{XRD}$ (图 1)显示: 针铁矿 (PDF\#81-0464) 、赤铁矿 (PDF\#79-1741) 、高岭石 (PDF\#79-1570) 在相应位置均有明显的特征峰, 没有出 现其他物相的衍射峰. 而根据谢乐方程, 利用 Jade 软件 计算了三种可变电荷矿物晶粒的平均大小, 结果分别为 针铁矿 $80.4 \mathrm{~nm}$ 、赤铁矿 $48.7 \mathrm{~nm}$ 、高岭石 $57.6 \mathrm{~nm}$. 综 上结果可知所用样品均为纳米级别高纯度矿物.

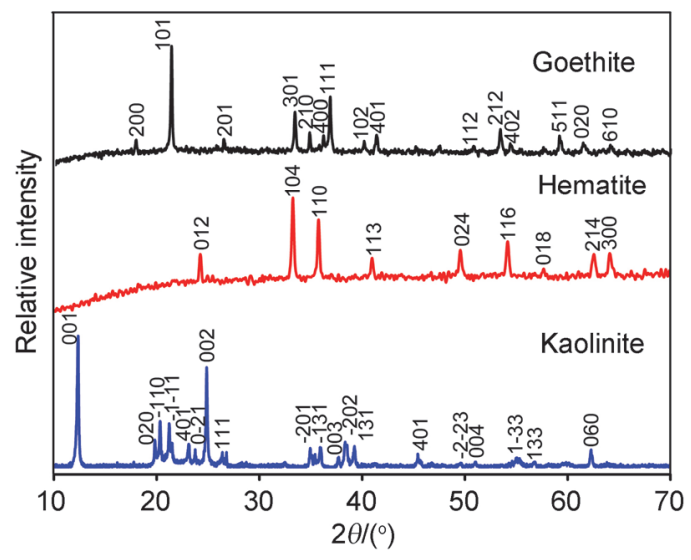

图 1 三种可变电荷矿物的 XRD 图

Figure 1 XRD results of three variable charge minerals

三种可变电荷矿物的 FTIR 结果见图 2, 对应红外吸 收峰列于表 1. 针铁矿在 $901 、 799 、 612 、 489 \mathrm{~cm}^{-1}$ 处出 现了特征吸收峰, 其中 901 和 $799 \mathrm{~cm}^{-1}$ 为 $\mathrm{Fe}-\mathrm{OH}$ 振动吸 收峰 ${ }^{[28,29]}, 612$ 和 $489 \mathrm{~cm}^{-1}$ 为 $\mathrm{Fe}-\mathrm{O}$ 振动吸收峰 ${ }^{[29,30]}$. 此 外, 针铁矿在 $1657 、 3118 、 3341 \mathrm{~cm}^{-1}$ 处的峰主要为 $-\mathrm{OH}$ 振动吸收峰, 其中 $3118 \mathrm{~cm}^{-1}$ 处属于水合羟基振动 ${ }^{[29,31]}$, 而 1657 和 $3341 \mathrm{~cm}^{-1}$ 主要为表面吸附 $\mathrm{H}_{2} \mathrm{O}$ 的弯曲和伸 缩振动吸收峰 ${ }^{[29,30]}$. 赤铁矿特征吸收峰与针铁矿相似, 
也在相近位置出现了 $\mathrm{Fe}-\mathrm{OH}\left(898 \text { 和 } 798 \mathrm{~cm}^{-1}\right)^{[28 ~ 31]}$ 、 $\mathrm{Fe}-\mathrm{O}\left(534 \text { 和 } 456 \mathrm{~cm}^{-1}\right)^{[29 \sim 31]}$ 振动吸收峰和表面吸附 $\mathrm{H}_{2} \mathrm{O}$ 的弯曲振动和伸缩振动吸收峰(分别为: 1634 和 3428 $\left.\mathrm{cm}^{-1}\right)^{[29,30,32]}$. 但与针铁矿相比, 赤铁矿的 $\mathrm{Fe}-\mathrm{OH}$ 振动吸 收峰非常弱. 这是由于针铁矿本身还有较多 $\mathrm{Fe}-\mathrm{OH}$, 因 此其吸收峰也较强. 高岭石的 FTIR 图显示了其在 $3620 \sim 3700 \mathrm{~cm}^{-1}$ 和 $400 \sim 1200 \mathrm{~cm}^{-1}$ 处含有典型的红外 特征吸收峰, 其中 $3623 \mathrm{~cm}^{-1}$ 处为四面体与八面体的层 间-OH 群振动峰, 3652 和 $3695 \mathrm{~cm}^{-1}$ 为表面 $-\mathrm{OH}$ 群振动 峰 ${ }^{[33,34]}$. 在 $400 \sim 1200 \mathrm{~cm}^{-1}$ 区域内主要为 $\mathrm{Si}-\mathrm{O} 、 \mathrm{Al}-\mathrm{OH}$ 振动吸收峰, 1111、1039、999 $\mathrm{cm}^{-1}$ 属于 $\mathrm{Si}-\mathrm{O}$ 振动; 915 $\mathrm{cm}^{-1}$ 归属为内部 Al-OH 振动; 789、749、698 $\mathrm{cm}^{-1}$ 属于 二氧化硅的 $\mathrm{Si}-\mathrm{O}$ 振动; $514 \mathrm{~cm}^{-1}$ 主要为 $\mathrm{Fe}-\mathrm{O}$ 或 $\mathrm{Si}-\mathrm{O}-\mathrm{Al}$ 振动; 465、420 $\mathrm{cm}^{-1}$ 则为 $\mathrm{Si}-\mathrm{O}-\mathrm{Si}$ 振动 ${ }^{[34]}$. 上述结果表 明针铁矿、赤铁矿、高岭石表面都具有羟基基团, 其质 子化、去质子化能力将直接决定矿物表面的酸碱性质.

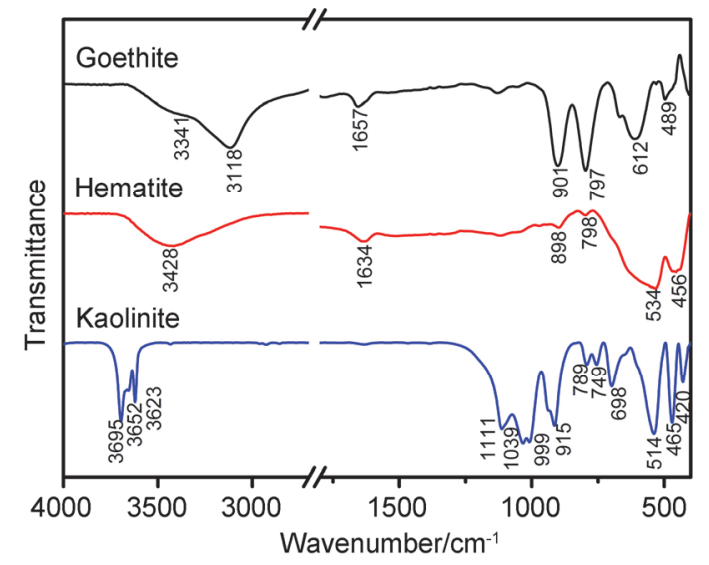

图 2 三种可变电荷矿物的 FTIR 图

Figure 2 FTIR results of three variable charge minerals

表 1 三种可变电荷矿物的红外吸收峰位置

Table 1 Infrared peak positions of three variable minerals

\begin{tabular}{llll}
\hline 样品 & 波数 $/ \mathrm{cm}^{-1}$ & 官能团 & 参考文献 \\
\hline 针铁矿 & 901,799 & $\mathrm{Fe}-\mathrm{OH}$ & {$[28,29]$} \\
& 612,489 & $\mathrm{Fe}-\mathrm{O}$ & {$[29,30]$} \\
& 1657,3341 & $\mathrm{H}-\mathrm{OH}$ & {$[29,30]$} \\
& 3118 & 水合-OH & {$[29,31]$} \\
赤铁矿 & 898,798 & $\mathrm{Fe}-\mathrm{OH}$ & {$[28 \sim 30]$} \\
& 534,456 & $\mathrm{Fe}-\mathrm{O}$ & {$[29,30,32]$} \\
& 1634,3428 & $\mathrm{H}-\mathrm{OH}$ & {$[29,30,32]$} \\
高岭石 & 3623 & 层间-OH & {$[33,34]$} \\
& 3652,3695 & 表面-OH & {$[33,34]$} \\
& $1111,1039,999$ & $\mathrm{Si}-\mathrm{O}$ & {$[33,34]$} \\
& 915 & 内部 $\mathrm{Al}-\mathrm{OH}$ & {$[33,34]$} \\
& $789,7499,698$ & $\mathrm{SiO} 2$ 的 $\mathrm{Si}-\mathrm{O}$ & {$[34]$} \\
& 514 & $\mathrm{Fe}-\mathrm{O}$ 或 $\mathrm{Si}-\mathrm{O}-\mathrm{Al}$ & {$[34]$} \\
& 465,420 & $\mathrm{Si}-\mathrm{O}-\mathrm{Si}$ & {$[34]$} \\
\hline
\end{tabular}

\section{2 酸碱电位滴定结果}

酸碱电位滴定实验中, 根据每次滴定平衡状态下的
表面可变电荷量 $Q$ 可以绘制滴定曲线, 获得表面电荷量 $Q$ 与体系 $\mathrm{pH}$ 的关系图. 如图 3 所示, 三种矿物在 0.1 、 0.3 和 $0.7 \mathrm{~mol} / \mathrm{L} \mathrm{NaCl}$ 溶液中的三条滴定曲线中表面电 荷量 $Q$ 随 $\mathrm{pH}$ 升高而明显降低, 说明了其表面有可变电 荷的存在. 3 条曲线有一共同交点被称为电荷零点, 其 所对应的 $\mathrm{pH}$ 记为 $\mathrm{pH}_{\mathrm{pzc}}$ (实验 $)^{[35]}$. 针铁矿、赤铁矿、高 岭石的 $\mathrm{pH}_{\mathrm{pzc}}$ (实验)分别为 $5.0 、 9.0$ 和 4.8 (表 2). 实验误 差 $\Delta \mathrm{pH} \approx \pm 0.2$.

三种可变电荷矿物在水溶液中均具有一定溶解性, 在极酸、极碱环境下溶解性更大, 表现为滴定曲线在 $\mathrm{pH}$ $=4$ 以下和 $\mathrm{pH}=8$ 以上呈现不同程度的突变现象. 并且, 在 $\mathrm{pH}=4$ 以上离子强度对表面电荷量的影响很小, 相似 的结果在 Szekeres 等 ${ }^{[36]}$ 的研究中也有发现. 此外实验获 得的针铁矿、高岭石在 $0.7 \mathrm{~mol} / \mathrm{L}$ 下的滴定曲线在 $\mathrm{pH}=$ 3.5 前还在上升, 说明在滴加 $\mathrm{NaOH}$ 溶液的最初阶段矿 物表面仍发生质子化作用. 这与 Gao 和 $\mathrm{Mucci}^{[21]}$ 的实验 结果是一致的, 而在其他两个较低的离子强度下并未出 现这种现象, 所以可能是过多 $\mathrm{Na}^{+}$竞争吸附和与矿物表 面正电荷的静电排斥作用所引起的结果. 而赤铁矿这种 现象并不太明显, 这可能与表面活性位点有关.

\section{3 应用 SCM 计算矿物表面酸碱性质参数}

\subsection{1 矿物表面活性位点浓度、密度计算}

矿物表面活性位点浓度 $H_{\mathrm{s}}$ 决定矿物表面 $\mathrm{H}^{+}$和 $\mathrm{OH}^{-}$ 的吸附容量, 是表示矿物酸碱缓冲能力的重要指标, 可 以根据 Gran 函数图获得 ${ }^{[37 ~ 39]}$. 由于矿物表面活性位点 具有两性, 在相应 $\mathrm{pH}$ 下可以发生质子化和去质子化反 应, 所以本研究采用了 1-site/2-p $K$ 表面络合模型分析矿 物表面活性位点的酸碱反应. 在用 $\mathrm{NaOH}$ 标准液滴加矿 物悬浮液的过程中, 矿物表面活性位点 $(\equiv \mathrm{SOH})$ 会发生 去质子化反应(如式 1 ):

$$
\equiv \mathrm{SOH}_{2}^{+} \stackrel{-\mathrm{H}^{+}}{\longrightarrow} \equiv \mathrm{SOH} \stackrel{-\mathrm{H}^{+}}{\longrightarrow} \equiv \mathrm{SO}^{-}
$$

以 $\mathrm{NaOH}$ 标准液滴加体积 $V_{\mathrm{b}}$ 为横坐标, 溶液中 $\mathrm{H}^{+}$、 $\mathrm{OH}^{-}$物质的量为纵坐标, 可绘制 Gran 函数图(如图 4). 两条 Gran 函数线与横坐标的交点分别是去质子化反应 的起点 $V_{\mathrm{e} 1}$ 和终点 $V_{\mathrm{e} 2}$, 二者的差值是矿物表面活性位点 完成去质子化反应所需 $\mathrm{NaOH}$ 体积. 所以 $H_{\mathrm{s}}\left(\mathrm{mol} \cdot \mathrm{kg}^{-1}\right)$ 可以通过公式(2)计算:

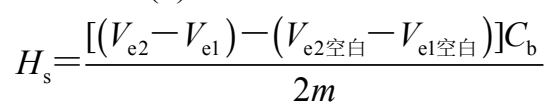

式中 $C_{\mathrm{b}}$ 为滴加 $\mathrm{NaOH}$ 的浓度, $m$ 为矿物的质量. 表面活 性位点密度 $D_{\mathrm{s}} /\left(\right.$ site・nm $\left.^{-2}\right)$ 代表胶体表面的-OH 量, 可以 通过公式(3)计算:

$$
D_{\mathrm{s}}=\frac{H_{\mathrm{s}} N_{\mathrm{A}}}{S_{\mathrm{BET}}}
$$

式中 $S_{\mathrm{BET}}$ 为矿物比表面积(表 2), $N_{\mathrm{A}}$ 是阿伏伽德罗常数 $6.02 \times 10^{23}$. 

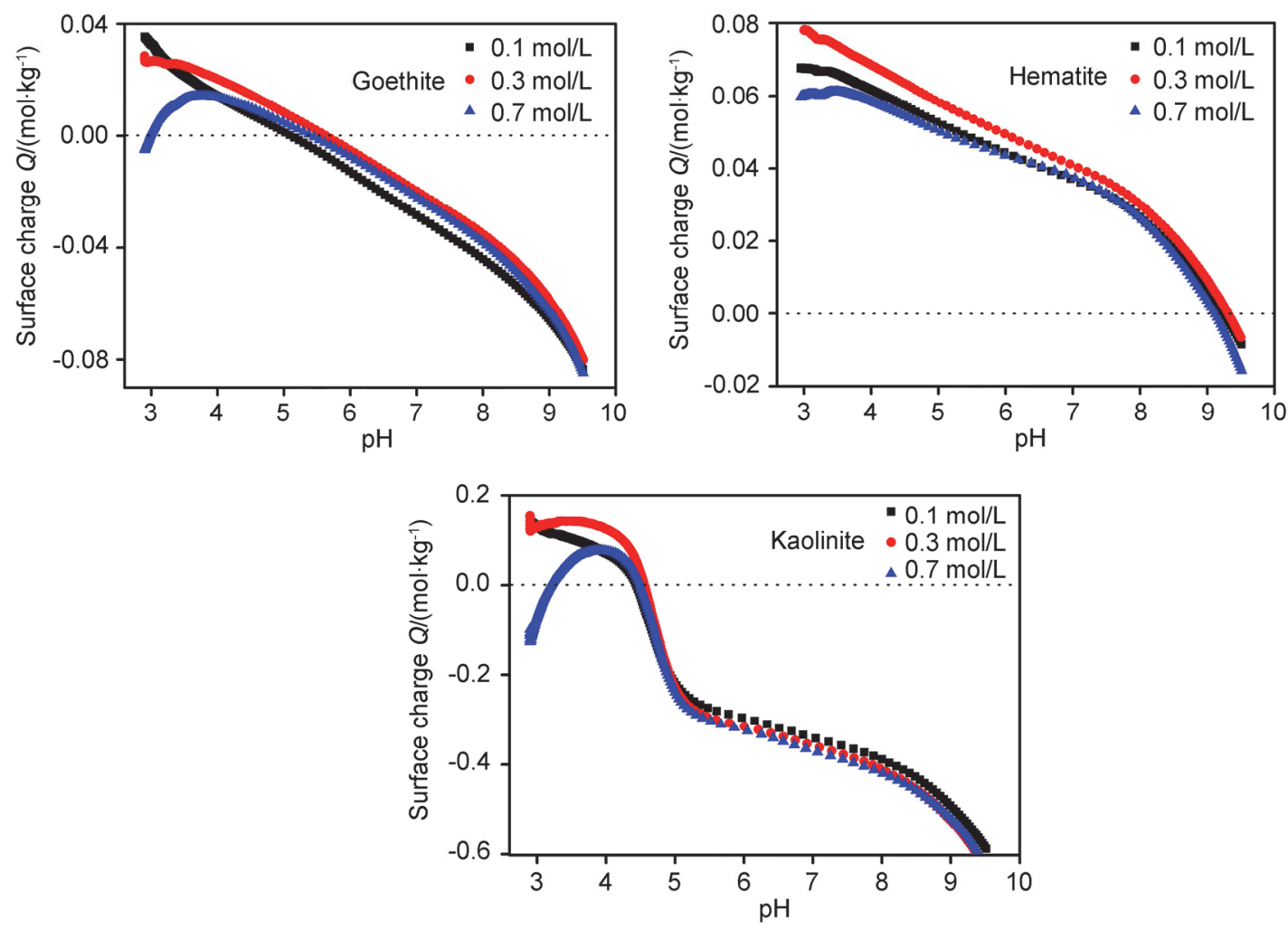

图 3 三种可变电荷矿物在不同离子强度溶液中的酸碱滴定曲线

Figure 3 The acid-base titration curves of three variable charge minerals in the solutions with various ionic strengths

表 2 三种可变电荷矿物以及土样的表面酸碱性质参数

Table 2 The surface acid-base properties of three variable charge minerals and soil

\begin{tabular}{cccccccc}
\hline 样品 & $H_{\mathrm{s}} /\left(\mathrm{mol} \cdot \mathrm{kg}^{-1}\right)$ & $S_{\mathrm{BET}} /\left(\mathrm{m}^{2} \cdot \mathrm{kg}^{-1}\right)$ & $D_{\mathrm{s}} /\left(\mathrm{site} \bullet \mathrm{nm}^{-2}\right)$ & $\mathrm{p} K_{\mathrm{a} 1}{ }^{\text {int }}$ & $\mathrm{p} K_{\mathrm{a} 2}{ }^{\text {int }}$ & $\mathrm{pH}_{\mathrm{pzc}}$ (计算 $)$ & $\mathrm{pH}_{\mathrm{pzc}}($ 实验 $)$ \\
\hline 针铁矿 & 0.0313 & $1.42 \times 10^{4}$ & 1.32 & 4.18 & 6.09 & 5.14 & $5.0 \pm 0.2$ \\
赤铁矿 & 0.0188 & $2.57 \times 10^{4}$ & 0.44 & 8.23 & 10.31 & 9.27 & $9.0 \pm 0.2$ \\
高岭土 & 0.0272 & $1.20 \times 10^{4}$ & 1.37 & 4.34 & 5.48 & 4.91 & $4.8 \pm 0.2$ \\
土壤 & 0.1313 & $3.50 \times 10^{4}$ & 2.26 & 3.17 & 6.33 & 4.70 & $4.8 \pm 0.2$ \\
\hline
\end{tabular}

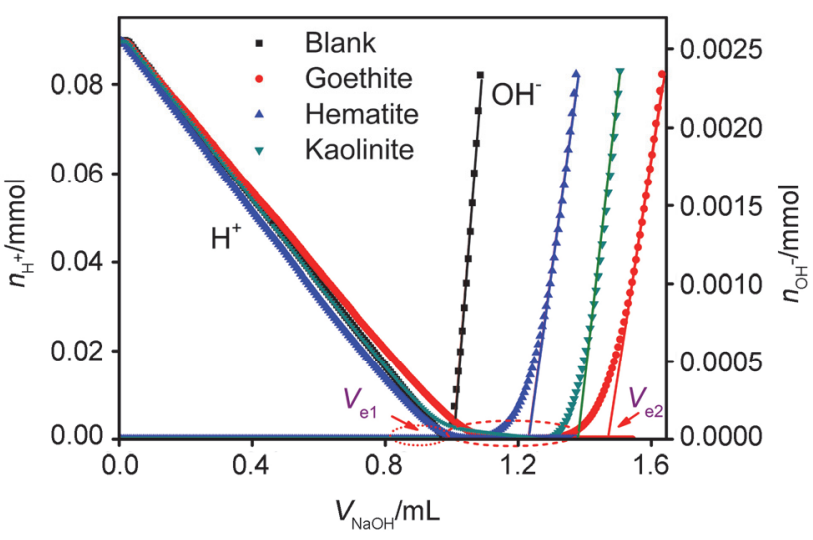

图 4 三种可变电荷矿物的 Gran 函数图

Figure 4 The Gran plot of three variable charge minerals

根据三种可变电荷矿物在 $0.1 \mathrm{~mol} / \mathrm{L} \mathrm{NaCl}$ 溶液中的 酸碱滴定数据绘制的 Gran 函数图(如图 4)所示. 空白 (Blank)是指未加入矿物的 $\mathrm{NaCl}$ 溶液, 可以看出单纯的
$\mathrm{NaCl}$ 溶液 $V_{\mathrm{e} 1} 、 V_{\mathrm{e} 2}$ 几乎重合, $\mathrm{NaOH}$ 溶液酸碱缓冲消耗 近似为零; 而三种可变电荷矿物的 $V_{\mathrm{e} 1}$ 与 $V_{\mathrm{e} 2}$ 之间存在不 同程度的差异，说明各种矿物在酸碱缓冲过程消耗了不 同体积的 $\mathrm{NaOH}$ 溶液. 依照公式(2)、(3)可以获得三种矿 物的 $H_{\mathrm{s}}$ 和 $D_{\mathrm{s}}$ (如表 2). 三种矿物表面位点浓度 $H_{\mathrm{s}}$ 大小 依次为: 针铁矿 $>$ 高岭石 $>$ 赤铁矿, 该结果直接反映了 土壤中的针铁矿、高岭石和赤铁矿的酸碱缓冲能力的强 弱.

针铁矿与赤铁矿相比, $S_{\mathrm{BET}}$ 虽然很小但却具有较大 的 $H_{\mathrm{s}}$, 这应与它们表面结构有关. 氧化铁结构中 1 个 $\mathrm{O}$ 可以与 $1 、 2$ 或 3 个 $\mathrm{Fe}$ 结合, 分别得到单配位体、双配 位体以及三配位体. 对应其表面 $\mathrm{Fe}-\mathrm{OH}$ 分别被称为 A、 C、B 型-OH. 第四种 D 型-OH 是指两个-OH 直接化学 吸附在裸露的 $\mathrm{Fe}^{3+}$. 一般认为 $\mathrm{C}$ 型 $-\mathrm{OH}$ 为惰性的, $\mathrm{A} 、 \mathrm{~B}$ 位点可以发生质子化和去质子化反应. 而第四种位点被 认为是路易斯酸位点, 只能释放质子 ${ }^{[40]}$. 在赤铁矿晶体 中，其优势晶面只含有 $\mathrm{C}$ 型惰性-OH，而针铁矿的大多 
数晶面虽也含有 $\mathrm{C}$ 型惰性- $\mathrm{OH}$ 但会有大量 $\mathrm{A}$ 型活性- $\mathrm{OH}$ 和少量 $\mathrm{D}$ 型 $-\mathrm{OH}$ 存在, 个别晶面还存在 $\mathrm{B}$ 型活性 $-\mathrm{OH}^{[41,42]}$. 所以, 针铁矿与赤铁矿相比, 活性晶面多, 表 面活性大. 由于高岭石表面含有 Al-OH、Si-OH、 $\mathrm{Al}_{2}-\mathrm{OH}$ 三种活性羟基, 溶液中的质子和氢氧根离子也会与高岭 石的层间-OH 发生反应 ${ }^{[20]}$, 因此实验所测高岭石的 $H_{\mathrm{s}}$ 也较高.

\subsection{2 矿物表面酸碱平衡常数与电荷零点计算}

根据 1-site/2-p $K$ 模型, 矿物表面活性位点存在质子 化和去质子化平衡. 在已知表面活性位点浓度 $H_{\mathrm{s}}$ 和每 个滴定平衡点的可变电荷量 $Q$ 时, 可以求算其酸碱平衡 常数, 如公式(4)和(5):

$$
\begin{aligned}
& \equiv \mathrm{SOH}+\mathrm{H}^{+} \leftrightarrow \equiv \mathrm{SOH}_{2}^{+} \\
& K_{\mathrm{aa}}=\frac{[\equiv \mathrm{SOH}]\left[\mathrm{H}^{+}\right]}{\left[\equiv \mathrm{SOH}_{2}^{+}\right]}=\frac{\left[H_{\mathrm{s}}-Q\right\}\left[\mathrm{H}^{+}\right]}{Q} \\
& \equiv \mathrm{SOH} \leftrightarrow \equiv \mathrm{SO}^{-}+\mathrm{H}^{+} \\
& K_{\mathrm{a} 2}=\frac{\left[\equiv \mathrm{SO}^{-}\right]\left[\mathrm{H}^{+}\right]}{[\equiv \mathrm{SOH}]}=\frac{Q\left[\mathrm{H}^{+}\right]}{\left[H_{\mathrm{s}}-Q\right]}
\end{aligned}
$$

式中表面物种浓度单位为 $\mathrm{mol} \cdot \mathrm{kg}^{-1}$. 假设 $\mathrm{pH}>\mathrm{pH}_{\mathrm{pzc}}$, $Q \approx\left[\equiv \mathrm{SO}^{-}\right]$; 假设 $\mathrm{pH}<\mathrm{pH}_{\mathrm{pzc}}, Q \approx\left[\equiv \mathrm{SOH}_{2}{ }^{+}\right]$. 通过直 线外推 $\mathrm{p} K_{\mathrm{a}}$ vs. $Q$ 曲线可获得两个固有酸碱平衡常数 $\mathrm{p} K_{\mathrm{a} 1}{ }^{\text {int }} 、 \mathrm{p} K_{\mathrm{a} 2}{ }^{\text {int: }}$

$$
\lim _{Q \rightarrow 0} \mathrm{p} K_{\mathrm{a}}=\mathrm{p} K_{\mathrm{a}}^{\text {int }}
$$

在电荷零点 $\mathrm{pH}_{\mathrm{pzc}}$ 时, $\left[\equiv \mathrm{SOH}_{2}{ }^{+}\right]=\left[\equiv \mathrm{SO}^{-}\right]$. 所以 $\mathrm{pH}_{\mathrm{pzc}}$ 也可以用 $\mathrm{p} K_{\mathrm{a} 1}{ }^{\text {int }} 、 \mathrm{p} K_{\mathrm{a} 2}{ }^{\text {int }}$ 表示:

$$
\mathrm{pH}_{\mathrm{pzc}}=0.5\left(\mathrm{p} K_{\mathrm{a} 1}^{\mathrm{int}}+\mathrm{p} K_{\mathrm{a} 2}^{\mathrm{int}}\right)
$$

三种矿物在 $0.1 \mathrm{~mol} / \mathrm{L} \mathrm{NaCl}$ 溶液中的 $\mathrm{p} K_{\mathrm{a}}$ vs. $Q$ 曲线 如图 5 所示. $\mathrm{p} K_{\mathrm{a} 1}{ }^{\text {int }} 、 \mathrm{p} K_{\mathrm{a} 2}{ }^{\mathrm{int}}$ 以及 $\mathrm{pH}_{\mathrm{pzc}}$ 计算结果列于表 2. 为区分两种方法所得 $\mathrm{pH}_{\mathrm{pzc}}$, 酸碱电位滴定曲线交点 对应 $\mathrm{pH}_{\mathrm{pzc}}$ 记为 $\mathrm{pH}_{\mathrm{pzc}}$ (实验), $\mathrm{SCM}$ 计算所得记为 $\mathrm{pH}_{\mathrm{pzc}}$ (计算). 二者数值非常接近, 说明 SCM 计算方法适用分 析氧化铁和高岭石表面酸碱性质. 此外, $\mathrm{pH}_{\mathrm{pzc}}$ 不随溶液 离子强度而变化, 是描述土壤胶体表面酸碱性质的重要 参数. 当土壤溶液 $\mathrm{pH}$ 低于 $\mathrm{pH}_{\mathrm{pzc}}$ 时, 胶体表面才会发生 质子化作用而缓冲酸化. 由图 5 及表 2 结果可知, 针铁 矿和高岭石的 $\mathrm{pH}_{\mathrm{pzc}}$ 较低(针铁矿 $\mathrm{pH}_{\mathrm{pzc}}=5.14$ 、高岭石 $\mathrm{pH}_{\mathrm{pzc}}=4.91$ ), 只有在重酸环境下才能吸附质子缓冲酸 化, 而赤铁矿的 $\mathrm{pH}_{\mathrm{pzc}}$ 较高 $\left(\mathrm{pH}_{\mathrm{pzc}}=9.27\right)$ 在一般环境下即 可. 所以就 $\mathrm{pH}_{\mathrm{pzc}}$ 而言, 赤铁矿相对于针铁矿、高岭石有 较好酸化缓冲效果.

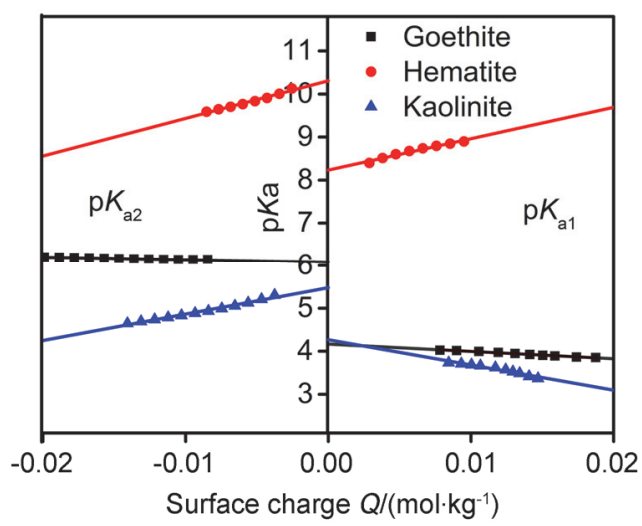

图 5 三种可变电荷矿物的酸碱平衡常数 $\mathrm{p} K_{\mathrm{a}}$ vs. $Q$ 曲线 Figure 5 The acid-base equilibrium constants $\mathrm{p} K_{\mathrm{a}}$ vs. $Q$ curves of three variable charge minerals

\section{4 模拟计算矿物表面化学物种}

为了深入解析影响矿物酸碱性质的表面化学物种, 本研究将 SCM 计算得到的 $H_{\mathrm{s}}$ 和 $\mathrm{p} K_{\mathrm{a}}^{\mathrm{int}}$ 带入 Visual Minteq 软件 ${ }^{[43 \sim 47]}$, 采用双电层表面络合模型(DLM-2pK) 模拟 计算不同 $\mathrm{pH}$ 下的矿物表面化学物种. DLM-2pK 模型认 为带有电荷的矿物表面吸引相邻液相的离子, 在矿物周 围形成带电荷的离子层, 它与矿物表面的电荷层一起构 成了双电层. 双电层模型 DLM 是土壤胶体水界面的离 子反应常用的经典表面络合模型 ${ }^{[26,43]}$. 因此, 本文亦采 用该模型进行矿物表面化学物种的模拟计算.

模拟结果如图 6 所示, 可以发现在低 $\mathrm{pH}$ 下，矿物表 面以 $\equiv \mathrm{SOH}_{2}{ }^{+}$为主, $\equiv \mathrm{SO}^{-}$、 $\equiv \mathrm{SOH}$ 逐渐吸附环境中质 子转变为 $\equiv \mathrm{SOH}_{2}{ }^{+}$, 这一过程应是可变电荷矿物缓冲酸 化的本质. 如果表面活性位点浓度较高, 则会有较多的 质子被吸附, 从而表现出较好的酸化缓冲能力. 在高 $\mathrm{pH}$ 下，矿物表面则以 $\equiv \mathrm{SO}^{-}$为主， $\equiv \mathrm{SOH}_{2}{ }^{+} 、 \equiv \mathrm{SOH}$ 逐 渐释放质子到环境中转变为 $\equiv \mathrm{SO}^{-}$, 这一过程能够缓冲 碱变化. $\equiv \mathrm{SOH}_{2}^{+}$、三 $\mathrm{SO}^{-}$浓度变化曲线交点处两者浓 度相同即为电荷零点 $\mathrm{pH}_{\mathrm{pzc}}$, 也是质子吸附与释放的分

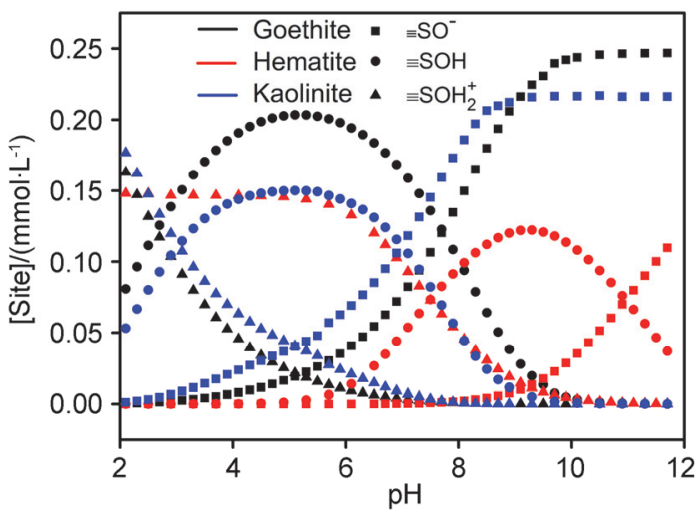

图 6 不同 $\mathrm{pH}$ 下可变电荷矿物表面化学物种分布

Figure 6 Chemical speciation distributions of three variable charge minerals at different $\mathrm{pH}$ 
界点. 当矿物 $\mathrm{pH}_{\mathrm{pzc}}$ 较低时, 很难吸附质子, 对缓冲环境 酸化效果较差, 但对缓冲碱化有较好效果. 高 $\mathrm{pH}_{\mathrm{pzc}}$ 矿 物则相反. 此外, 从模拟图发现在 $\equiv \mathrm{SOH}$ 与 $\equiv \mathrm{SOH}_{2}{ }^{+}$、 $\equiv \mathrm{SO}^{-}$的两个交点附近表面物种变化比较活跃, 所以当 环境 $\mathrm{pH}$ 接近 $\mathrm{p} K_{\mathrm{a} 1}{ }^{\text {int }} 、 \mathrm{p} K_{\mathrm{a} 2}{ }^{\text {int }}$ 时, 质子吸附与释放反应比 较剧烈. 综上所述, 表面物种的模拟较好地解释了酸碱 性质参数 $H_{\mathrm{s}} 、 \mathrm{pH}_{\mathrm{pzc}} 、 \mathrm{p} K_{\mathrm{a} 1}{ }^{\mathrm{int}} 、 \mathrm{p} K_{\mathrm{a} 2}{ }^{\text {int }}$ 对表达矿物酸碱缓 冲能力的意义.

\section{5 电位表征矿物表面质子化过程}

为了更好地表征三种矿物的表面质子化和去质子 化的过程, 我们测定了这三种矿物的开路电位随 $\mathrm{pH}$ 的 变化曲线 ${ }^{[48]}$. 从图 7 中可以看出, 三种矿物的开路电位 均随溶液 $\mathrm{pH}$ 升高而降低, 说明矿物表面化学物种在 $\mathrm{pH}$ 升高时均发生了质子脱附过程. 由其变化斜率可知三种 矿物的质子化程度: 针铁矿 $>$ 高岭石 $>$ 赤铁矿, 这与我 们基于酸碱电位滴定实验和 SCM 模型所估算的三种矿 物的缓冲能力大小是一致的, 均说明表面位点浓度越 大，质子化程度越大，矿物表面的缓冲能力越大.

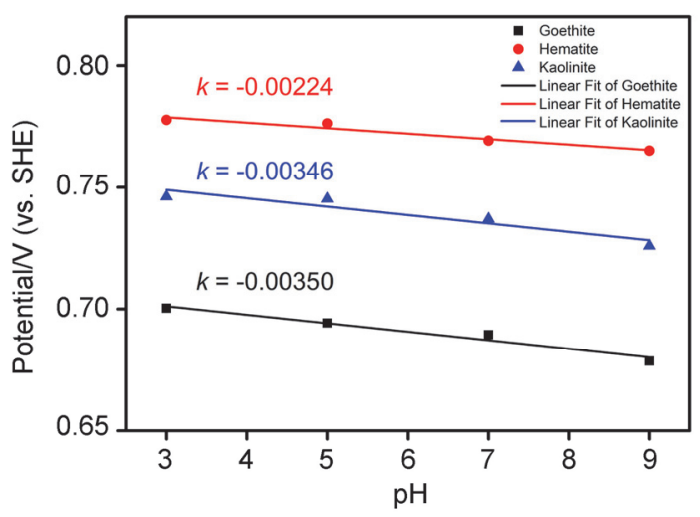

图 7 三种可变电荷矿物开路电位随 $\mathrm{pH}$ 变化曲线

Figure 7 The open circuit potentials of three variable charge minerals as a function of $\mathrm{pH}$

\section{6 红壤酸碱性质测试与表面物种分析}

上述测试结果主要针对红壤中的主要矿物, 我们进 一步尝试将该测试方法与表面络合模型应用于实际林 地红壤样品的表面酸碱性质分析. X 射线衍射(如图 8a) 及半定量分析结果(如表 3) 显示, 土样成分以高岭石 (PDF\#78-1996)为主, 含有部分二氧化硅(PDF\#79-1910) 和斜绿泥石 (PDF\#79-1270), 还有少量的针铁矿 (PDF\#81-0462)及微量的赤铁矿(PDF\#89-0599). X 射线 荧光光谱结果(如表 4)显示, 土样中除硅铝元素外, 还含 有较多的铁元素及少量其他元素. 根据酸碱电位滴定法 和 SCM 计算获得的两个 $\mathrm{pH}_{\mathrm{pzc}}$ 相近(如图 $8 \mathrm{~b}$ 和表 2), 说 明该方法亦适用于林地红壤表面酸碱性质. 土样的 $\mathrm{pH}_{\mathrm{pzc}}$ (4.70)略低于高岭土 $\mathrm{pH}_{\mathrm{pzc}}$ (4.91). 这可能是由于土 样中 $\mathrm{SiO}_{2}$ 的 $\mathrm{pH}_{\mathrm{pzc}}$ 较低所导致, 据文献报道 $\mathrm{SiO}_{2}$ 的 $\mathrm{pH}_{\mathrm{pzc}}$ 在 $1 \sim 3$ 范围内 ${ }^{[49]}$. $\mathrm{SiO}_{2}$ 的 $\mathrm{pH}_{\mathrm{pzc}}$ 虽然较低, 但研究发现
其在 $\mathrm{pH} 2 \sim 6$ 的范围内表面负电荷并不多 ${ }^{[50]}$. 在通常可 变电荷土壤 $\mathrm{pH}$ 范围内大部分是以不带电荷的 $\equiv \mathrm{SiOH}$ 存在, 只有少部分带负电荷的 $\equiv \mathrm{SiO}^{-}$. 所以 $\mathrm{SiO}_{2}$ 与高岭 土、铁氧化物相比在可变电荷土壤中的重要性较小 ${ }^{[51]}$. 氧化铁中针铁矿的缓冲能力较强, 提高了红壤的缓冲能 力, 而赤铁矿具有较高的 $\mathrm{pH}_{\mathrm{pzc}}$ 会提高土样的 $\mathrm{pH}_{\mathrm{pzc}}$, 是 可变电荷土壤中非常重要的矿物. 但在本土壤样品中铁 氧化物含量相比高岭石低很多, 所以其 $\mathrm{pH}_{\mathrm{pzc}}$ 更多由高 岭石决定.

表 3 XRD 定量分析红壤样品矿物组成

Table 3 Quantitative analyses of the red soil by X-ray diffraction spectroscopy

\begin{tabular}{cccccc}
\hline $\begin{array}{c}\text { 矿物 } \\
\text { Minerals }\end{array}$ & $\begin{array}{c}\text { 高岭石 } \\
\text { Kaolinite }\end{array}$ & $\begin{array}{c}\text { 石英 } \\
\text { Quartz }\end{array}$ & $\begin{array}{c}\text { 斜绿泥石 } \\
\text { Clinochlore }\end{array}$ & $\begin{array}{c}\text { 针铁矿 } \\
\text { Goethite }\end{array}$ & $\begin{array}{c}\text { 赤铁矿 } \\
\text { Hematite }\end{array}$ \\
\hline 含量 $/ \mathrm{wt} \%$ & 61.1 & 21.7 & 13.9 & 3.3 & $<0.1$ \\
\hline
\end{tabular}

表 $4 X$ 射线苂光光谱所测红壤样品元素组成

Table 4 Major elemental composition of the red soil sample measured by X-ray fluorescence spectrometry

\begin{tabular}{ccccccc}
\hline 元素 & $\mathrm{Si}$ & $\mathrm{Al}$ & $\mathrm{Fe}$ & $\mathrm{Ti}$ & $\mathrm{K}$ & $\mathrm{Mg}$ \\
\hline 含量 $/ \mathrm{wt} \%$ & 20.21 & 15.41 & 4.99 & 0.83 & 0.65 & 0.12 \\
\hline
\end{tabular}

\section{3 结论}

本文以酸碱电位滴定实验为基础结合表面络合模 型 SCM 研究了红壤中可变电荷矿物的酸碱缓冲能力. 研究结果表明: 应用 $\mathrm{SCM}$ 模型计算所得到的 $\mathrm{pH}_{\mathrm{pzc}}$ 与酸 碱电位滴定所得一致, 说明 SCM 能较好地适用于分析 针铁矿、赤铁矿及高岭石的表面酸碱性质; 所获得参数 结果表明, 针铁矿、高岭石表面活性位点浓度 $H_{\mathrm{s}}$ 较高, 说明其对酸化具有较好的缓冲效果. 但考虑到电荷零点 $\mathrm{pH}_{\mathrm{pzc}}$, 针铁矿、高岭石偏低在一般酸化环境下很难吸附 质子缓冲酸化. 所以在土壤矿物中赤铁矿可能发挥主要 的酸化缓冲作用. 这对我国不同母质红壤酸碱缓冲能力 的判定提供依据. 另外, 结合表面化学物种模拟分析, 进一步阐明酸化缓冲机制. 此外, 本研究将可变电荷矿 物 SCM 分析方法扩展到林地红壤, 结果同样适用, 这 或许可以为我国红壤酸碱缓冲能力测定方法的建立提 供依据.

\section{4 实验部分}

\section{1 样品和试剂}

实验所用针铁矿 $(30 \% \sim 63 \% \mathrm{Fe})$ 购自 Aldrich，赤铁 矿 $(99 \%)$ 购自 Alfa Aesar，高岭土采自广东省茂名市山阁 镇飞鼠岭. 邻苯二甲酸氢钾、碳酸钠、氢氧化钠、浓盐 酸和氯化钠均为分析纯试剂, 用超纯水(电阻 18.2 $\mathrm{M} \Omega \cdot \mathrm{cm})$ 配制各种溶液. 林地土壤采自广州市天河区华 南农业大学树木园 $\left(N 23^{\circ} 9^{\prime} 21^{\prime \prime}, E 113^{\circ} 21^{\prime} 16^{\prime \prime}\right)$, 为 古生代至中生代黑云母花岗岩发育而来的黏质高岭石 

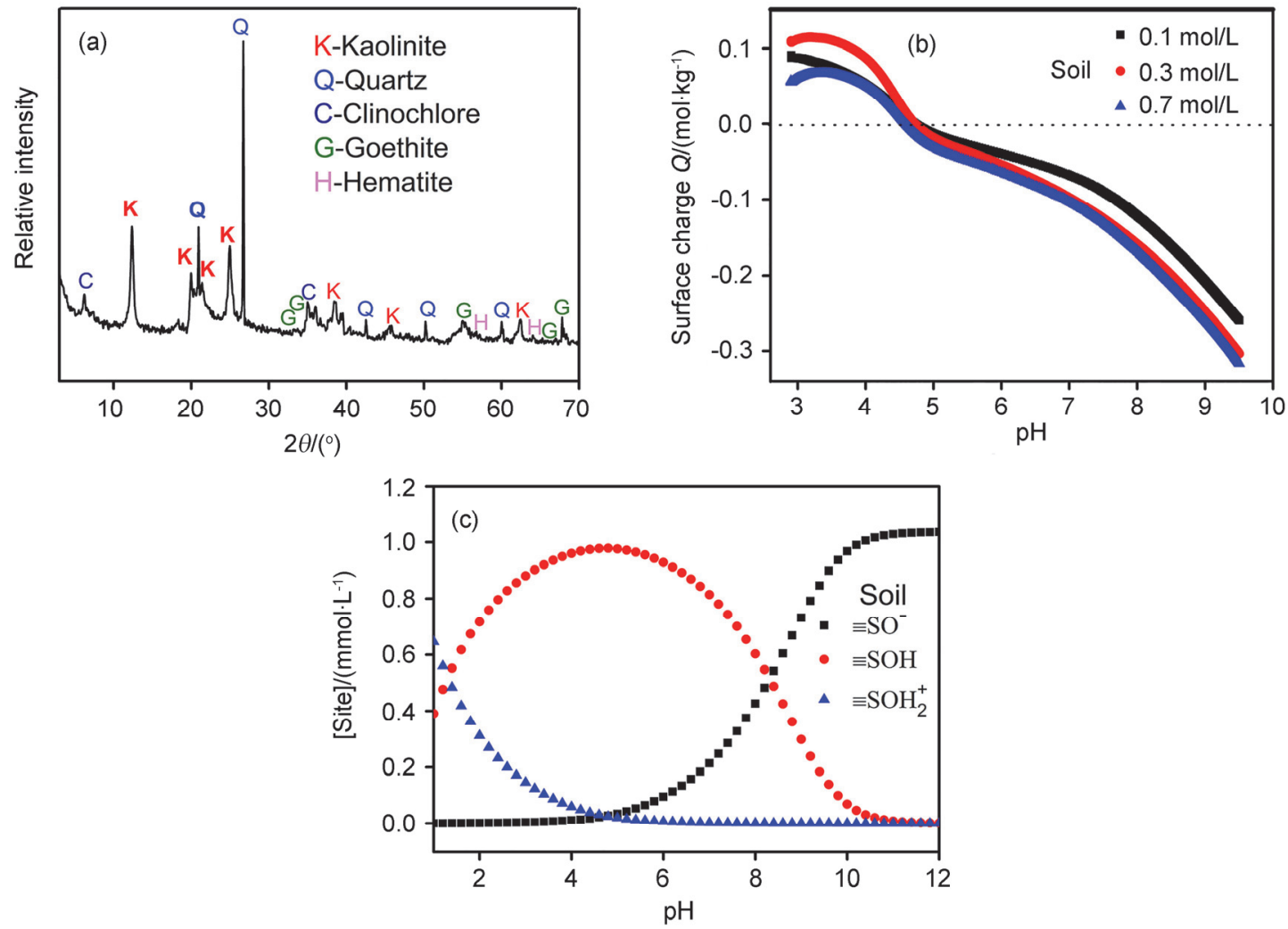

图 8 (a) 土样 $\mathrm{X}$ 射线衍射光谱及物相鉴定; (b) 土样酸碱滴定曲线; (c) 在不同 $\mathrm{pH}$ 下土样表面物种分布

Figure 8 (a) The X-ray diffraction spectroscopy and phase identification of the soil sample; (b) The acid-base titration curves of the soil sample; (c) The speciation diagrams of three variable charge minerals at different $\mathrm{pH}$

型酸性高热性-腐殖黏化湿润富铁土. 土壤一些基本理 化性质: $\mathrm{pH}$ 值 4.2 , 有机碳含量 $21.9 \mathrm{~g} \cdot \mathrm{kg}^{-1}$, 全磷 0.29 $\mathrm{g} \cdot \mathrm{kg}^{-1}$, 全氮 $4.8 \mathrm{~g} \cdot \mathrm{kg}^{-1}$, 全钾 $4.8 \mathrm{~g} \cdot \mathrm{kg}^{-1}$, 阳离子交换量 $44.9 \mathrm{cmol} \cdot \mathrm{kg}^{-1}$, 盐基饱和度 $3.9 \%$, 铝饱和度 $87.9 \%$, 游 离 $\mathrm{Fe}_{2} \mathrm{O}_{3} 44.6 \mathrm{~g} \cdot \mathrm{kg}^{-1}$, 铁游离度 $70.1 \%$. 土样自然风干后 过 200 目篮子备用.

\section{2 样品表征}

样品由德国 Bruker 公司的 D8 advance X 射线衍射 仪、Vertex 70 傅里叶红外光谱仪进行表征. 比表面积测 定实验在美国 Micromeritics 公司的 ASAP 2020 上进行. $X$ 射线苂光光谱在荷兰帕纳科公司的 AxiosPW4400 菼 光光谱仪上完成.

\section{3 酸碱电位滴定实验}

酸碱电位滴定实验在瑞士万通全自动电位滴定仪 (Metrohm, 905Titrando)上进行. 滴定结果会受 $\mathrm{CO}_{2}$ 、环 境温度、溶解性以及溶液离子强度等因素影响. 因为 $\mathrm{CO}_{2}$ 会吸附在可变电荷矿物表面而提高表面负电荷量从 而影响实验结果. 因此本实验整个过程保持 $\mathrm{N}_{2}$ 持续通 入以排除 $\mathrm{CO}_{2}$ 的干扰. 实验环境温度保持在 $25{ }^{\circ} \mathrm{C}$ 左右 以排斥温度对水的电离常数、表面质子吸附的影响. 滴 定实验在 $0.1 、 0.3$ 和 $0.7 \mathrm{~mol} / \mathrm{L}$ 三种离子强度的 $\mathrm{NaCl}$ 溶 液中进行. 称取 $0.56 \mathrm{~g}$ 的样品, 加入 $70 \mathrm{~mL}$ 的 $\mathrm{NaCl}$ 溶液,
持续摚拌, 约 $1 \mathrm{~h}$ 稳定. 然后使用全自动电位滴定仪测 定悬浊液的 $\mathrm{pH}$ 变化, 具体滴定步骤为: 先用 $0.1 \mathrm{~mol} / \mathrm{L}$ 的 $\mathrm{HCl}$ 溶液滴定至 $\mathrm{pH}=2.9$ (一次性加 $0.5 \mathrm{~mL}$, 搅拌 10 $\min$ 后再缓慢滴定), 再用 $0.1 \mathrm{~mol} / \mathrm{L}$ 的 $\mathrm{NaOH}$ 溶液滴定 至 $\mathrm{pH}=9.5$. 每次滴定前, 用 4.0、7.0、9.0 的 $\mathrm{pH}$ 缓冲液 矫正电极, 并标定 $\mathrm{HCl} 、 \mathrm{NaOH}$ 标准液的浓度. 滴定速度 为每 20 秒 $0.6 \mu \mathrm{L}$, 以保证每步滴定都能达到酸碱平衡, 整个实验过程中持续通入 $\mathrm{N}_{2}$ 以排除 $\mathrm{CO}_{2}$ 干扰. 在酸碱 滴定实验过程中, 土壤表面电荷不断变化. 每个滴定平 衡点样品的表面可变电荷量 $Q$ 可以通过酸、碱的加入量 以及溶液中 $\mathrm{H}^{+} 、 \mathrm{OH}^{-}$平衡浓度计算，计算公式如下：

$$
Q=\frac{\left(C_{\mathrm{a}}-\mathrm{C}_{\mathrm{b}}\right)+\left(\left[\mathrm{OH}^{-}\right]-\left[\mathrm{H}^{+}\right]\right)}{a}
$$

式中 $Q$ 单位是 $\mathrm{mol} \cdot \mathrm{kg}^{-1}, a$ 为矿物浓度 $\mathrm{kg} \cdot \mathrm{L}^{-1}$.

\section{4 矿物表面开路电位实验}

矿物表面开路电位实验在上海辰华 CHI660C 电化 学工作站上进行. 采用内径为 $3 \mathrm{~mm}$ 的玻碳电极 $\mathrm{GC}$ 作 为矿物电极载体, 实验前先用砂纸和 $\gamma-\mathrm{Al}_{2} \mathrm{O}_{3}$ 抛光粉 (1 $\mu \mathrm{m} 、 0.05 \mu \mathrm{m}$ )打磨去除电极表面杂质, 并依次在 $1: 1$ $\mathrm{HNO}_{3}$ 水溶液、 $1: 1$ 乙醇水溶液、超纯水中超声清洗 2 $3 \mathrm{~min}$. 将 $2 \mathrm{mg}$ 矿物粉末溶解在 $500 \mu \mathrm{L} 0.5 \mathrm{wt} \%$ Nafion 
溶液中, 超声 $1 \mathrm{~h}$, 至分散均匀的悬浊液. 使用微量注射 器取 $2 \mu \mathrm{L}$ 悬浊液涂覆在处理好的 $\mathrm{GC}$ 电极载体上, 并在 空气中风干 $30 \mathrm{~min}$, 制成矿物电极以备用. 开路电位测 量在三电极体系中进行, 矿物电极作为工作电极, 饱和 甘采电极作为参比电极 $\left(+0.2443 \mathrm{mV}\right.$ vs SHE, $\left.25{ }^{\circ} \mathrm{C}\right)$, 铂电极作为对电极, 将矿物电极依次放在含有 $0.1 \mathrm{~mol} / \mathrm{L}$ $\mathrm{NaCl}$ 溶液的不同 $\mathrm{pH}(\mathrm{pH}=3,5,7,9)$ 下, 测定其开路电 位, 直到电位变化趋于稳定(小于 $0.5 \mathrm{mV} / \mathrm{min}$ ), 平衡时 间大约 $30 \mathrm{~min}$.

\section{References}

[1] Li, Q. K. Chinese Red Soil, Vol. 1 2, Eds.: Zhao, Q. G.; Shi, H.; Gong, Z. T., Science Press, Beijing, 1983, p. 1 (in Chinese). (李庆 迬, 中国红壤, 卷 $1 \sim 2$, 编辑: 赵其国, 石华, 龚子同, 科学出版 社, 北京, 1983, p. 1.)

[2] Xiong, Y.; Li, Q. K. Chinese Soil, Science Press, Beijing, 1990, pp. 502 508 (in Chinese). (熊毅, 李庆達, 中国土壤, 科学出版社, 北京, 1990, pp. 502 508.)

[3] Brown, K. A. Water, Air, Soil Pollut. 1987, 32, 201.

[4] Liao, B.; Guo, Z.; Zeng, Q.; Probst, A.; Probst, J. Water, Air, Soil Pollut.: Focus 2007, 7, 151.

[5] Fu, L.; Wu, J.; Yang, Y.; Qiu, L. Environ. Sci. 1993, 14(1), 20 (in Chinese). (傅柳松, 吴杰民, 杨影, 邱理均, 环境科学, 1993, 14(1), 20.)

[6] Zhao, Y.; Duan, L.; Xing, J.; Larssen, T.; Nielsen, C. P.; Hao, J. Environ. Sci. Technol. 2009, 43, 8021.

[7] Guo, J. H.; Liu, X. J.; Zhang, Y.; Shen, J. L.; Han, W. X.; Zhang, W. F.; Christie, P.; Goulding, K. W. T.; Vitousek, P. M.; Zhang, F. S. Science 2010, 327, 1008.

[8] Liao, B. H.; Dai, Z. H. Acta Sci. Circumstantiae 1991, 11, 425 (in Chinese). (廖柏寒, 戴昭华, 环境科学学报, 1991, 11, 425.)

[9] Reuss, J.; Cosby, B.; Wright, R. Nature 1987, 329, 27.

[10] Wright, R.; Cosby, B.; Flaten, M.; Reuss, J. Nature 1990, 343, 53.

[11] Larssen, T.; Schnoor, J. L.; Seip, H. M.; Dawei, Z. Sci. Total Environ. 2000, 246, 175.

[12] Li, J. Y.; Wang, N.; Xu, R. K. Soils 2009, 41, 932 (in Chinese). (李 九玉, 王宁, 徐仁扣, 土壤, 2009, 41, 932.)

[13] Xu, R. K. Soils 2015, 47, 238 (in Chinese). (徐仁扣, 土壤, 2015, 47, 238.)

[14] Alekseeva, T.; Alekseev, A.; Xu, R. K.; Zhao, A. Z.; Kalinin, P. Environ. Geochem. Health 2011, 33, 137.

[15] Dixon, J. B.; Weed, S. B.; Dinauer, R. C. Minerals in Soil Environments, 2nd ed., Eds.: Barnhisel, R. I.; Bertsch, P. M., SSSA, USA, 1989, Chapter 15, p. 729

[16] Yu, T. R.; Chen, Z. C. The Chemical Process in the Soil, Vol. 14, Ed.: Chen, Z. C., Science Press, Beijing, 1990, p. 432 (in Chinese). (于天仁, 陈志诚, 土壤发生中的化学过程, 卷 14, 编辑: 陈志诚, 科学出版社, 北京, 1990, p. 432.)

[17] Yu, T. R.; Wang, Z. Q. Soil Analytical Chemistry, Vol. 11, Eds.: Chen, J. F.; He, Q., Science Press, Beijing, 1987, p. 337 (in Chinese). (于天仁, 王振权, 土壤分析化学, 卷 11 , 编辑: 陈家坊, 何 群, 科学出版社, 北京, 1987, p. 337.)

[18] Wang, X. G.; Li, F. B. Persistent Organic Pollutants Forum and National Symposium on Persistent Organic Pollutants, Eds.: Yu, G.; Huang, J.; Wang, B.; Liu, Y. C., Chinese Chemical Society, Beijing, 2006, pp. 215 223 (in Chinese). (王旭刚, 李芳柏, 持久性有机污 染物论坛暨持久性有机污染物全国学术研讨会, 编者: 余刚, 黄 俊, 王斌, 刘意成, 中国化学会, 北京, 2006, pp. 215 223.)

[19] Wang, X. G.; Sun, L. R.; Zeng, F.; Li, F. B. Res. Environ. Sci. 2009, 22(4), 60 (in Chinese). (王旭刚, 孙丽蓉, 曾芳, 李芳柏, 环境科 学研究, 2009, 22(4), 60.)
[20] Gao, S.; He, G. P.; Wu, H. H.; Sun, W. Y. Acta Petrol. Mineral. 2005 24, 239 (in Chinese). (高嵩, 何广平, 吴宏海, 孙伟亚, 岩石矿物 学杂志, 2005, 24, 239.)

[21] Gao, Y.; Mucci, A. Geochim. Cosmochim. Acta 2001, 65, 2361.

[22] Tan, W. F; Zhou, S. Z.; Liu, F.; Feng, X. H.; Li, X. H. Soils 2007, 39(5), 726 (in Chinese). (谭文峰, 周素珍, 刘凡, 冯雄汉, 李学垣, 土壤, 2007, 39(5), 726.)

[23] Xu, R. K.; Zhao, A. Z.; Jiang, J. Ecol. Environ. 2011, 20(10), 1395 (in Chinese). (徐仁扣, 赵安珍, 姜军, 生态环境学报, 2011, 20(10), 1395.)

[24] Yu, X. J.; Chou, R. L. Chongqing Environ. Sci. 1998, 20(3), 11 (in Chinese). (于锡军, 仇荣亮, 重庆环境科学, 1998, 20(3), 11.)

[25] Stumm, W. Chemistry of the Solid-water Interface: Processes at the Mineral-water and Particle-water Interface in Natural Systems, John Wiley \& Son Inc., New York, 1992. pp. 13 23.

[26] Tombácz, E.; Szekeres, M. Langmuir 2001, 17, 1411.

[27] Davis, J. A.; Leckie, J. O. J. Colloid Interface Sci. 1978, 67, 90.

[28] Cagnasso, M.; Boero, V.; Franchini, M. A.; Chorover, J. Colloids Surf., $B$ 2010, 76,456 .

[29] Liu, T.; Li, X.; Li, F.; Zhang, W.; Chen, M.; Zhou, S. Colloids Surf., A 2011, 379(1), 143.

[30] Li, X.; Liu, T.; Li, F.; Zhang, W.; Zhou, S.; Li, Y. J. Soil. Sediment. 2012, 12(2), 217.

[31] Zhou, D. H.; Li, X. H.; Xu, F. L. J. Huazhong Agric. Univ. 1996, 15(2), 153 (in Chinese). (周代华, 李学垣, 徐风琳, 华中农业大学 学报, 1996, 15(2), 153.)

[32] Liu, T.; Li, X.; Li, F.; Tao, L.; Liu, H. Soil Sci. 2014, 179, 468.

[33] Djomgoue, P.; Njopwouo, D. J. Surf. Eng. Mater. Adv. Technol. 2013, 3, 275 .

[34] Saikia, B. J.; Parthasarathy, G. J. Mod. Phys. 2010, 1, 206.

[35] Lu, S. J.; Tan, W. F.; Liu, F.; Feng, X. H. Acta Pedol. Sin. 2006 43(5), 756 (in Chinese). (陆泗进, 谭文峰, 刘凡, 冯雄汉, 土壤学 报, 2006, 43(5),756.)

[36] Szekeres, M.; Tombácz, E. Colloids Surf., A 2012, 414, 302.

[37] Jolsterå, R.; Gunneriusson, L.; Forsling, W. J. Colloid Interface Sci. 2010, 342, 493.

[38] Frini-Srasra, N.; Kriaa, A.; Srasra, E. Russ. J. Electrochem. 2007, $43,795$.

[39] Wu, Z. S.; Zhang, W. M.; Sun, Z. X. Acta Chim. Sinica 2010, 68(8), 769 (in Chinese). (吴震生, 张卫民，孙中溪，化学学报，2010, 68(8), 769.)

[40] Davis, J. A.; Kent, D. Rev. Mineral. Geochem. 1990, 23, 177.

[41] Kubicki, J. D.; Paul, K. W.; Kabalan, L.; Zhu, Q.; Mrozik, M. K.; Aryanpour, M.; Pierre-Louis, A. M.; Strongin, D. R. Langmuir 2012, 8, 14573.

[42] Cornell, R. M.; Schwertmann, U. The Iron Oxides: Structure, Properties, Reactions, Occurrences and Uses, John Wiley \& Sons, 2003, pp. 221 223.

[43] Pagnanelli, F.; Bornoroni, L.; Toro, L. Environ. Sci. Technol. 2004, $38,5443$.

[44] Liu, T.; Li, X.; Waite, T. D. Environ. Sci. Technol. 2014, 48, 14564.

[45] Liu, T.; Li, X.; Waite, T. D. Environ. Sci. Technol. 2013, 47(13), 7350.

[46] Liu, T.; Li, X.; Waite, T. D. Environ. Sci. Technol. 2013, 47(23), 13712.

[47] Liu, T.; Li, X.; Zhang, W.; Hu, M.; Li, F. J. Colloid Interface Sci. 2014, $423,25$.

[48] Yanina, S. V.; Rosso, K. M. Science 2008, 320(5873), 218.

[49] Janusz, W.; Skwarek, E.; Zarko, V. I.; Gun'ko, V. M. Physicochem. Probl. Miner. Process. 2007, 41, 215.

[50] Pyman, M.; Bowden, J.; Posner, A. Soil Res. 1979, 17, 191.

[51] Yu, T. R.; Ji, G. L.; Ding, C. P. Electrochemical Behavior of Variable Charge Soils, Vol. 2, Eds.: Yu, T. R.; Zhao, A. Z., Science Press, Beijing, 1996, p. 9 (in Chinese). (于天仁, 季国亮, 丁昌璞, 可变 电荷土壤的电化学, 卷 2 , 编辑: 于天仁, 赵安珍, 科学出版社, 北京, 1996, p. 9.) 